\title{
Injury Assessment for Physics-Based Characters
}

\author{
Thomas Geijtenbeek, Diana Vasilescu, and Arjan Egges \\ Games and Virtual Worlds, Utrecht University, The Netherlands
}

\begin{abstract}
Determining injury levels for virtual characters is an important aspect of many games. For characters that are animated using simulated physics, it is possible assess injury levels based on physical properties, such as accelerations and forces. We have constructed a model for injury assessment that relates results from research on human injury response to parameters in physics-based animation systems. We describe a set of different normalized injury measures for individual body parts, which can be combined into a single measure for total injury. Our research includes a user study in which human observers rate the injury levels of physics-based characters falling from varying heights at different orientations. Results show that the correlation between our model output and perceived injury is stronger than the correlation between perceived injury and fall height (0.603 versus 0.466 , respectively, with $N=1020$ and $p<0.001)$.
\end{abstract}

\section{Introduction}

Severe physical trauma is a common ordeal for many virtual game characters. In fact, many games are designed around the concept of inflicting as much injury as possible on other characters. When such games aim for high realism, it is important that the assessment of injury is accurate.

In games that use kinematic animation systems, the possibilities for assessment are limited, because there exists no direct relation to knowledge on human injury. An example indicator could be the initial height of a character that is falling to the ground, but such a measure ignores the specific forces acting on the character during impact. In physics-based animation systems, all animation is the result of simulated physics. Specific physics-based parameters can be used to estimate injury in a way that is in line with physical reality.

There exist several games that model physical injury of physics-based characters. An example is Stair Dismount, a game in which the goal is to inflict as much damage as possible on a character by pushing it from a staircase. However, there exist no publications that explain what parameters and thresholds are used as a basis for these models, nor are there any published studies that show if and how such models correlate with perceived injury levels.

We propose an injury assessment model for physics-based virtual characters that is based on a comprehensive set of publications on human injury response levels. Our model produces a set of normalized measures that represent injury levels for several individual body parts. No tuning is required, as all parameters 
are directly derived from publications on injury research. Normalization enables straightforward combination of individual measures into a single measure representing total injury. Our research includes a user study in which the output of our injury assessment model is compared to injury levels as perceived by human observers.

We believe there are several uses for our model. First, it can be used to measure injury of in-game physics-based characters; either to keep track of the overall health of the character, or to monitor the condition of specific body parts. Furthermore, we feel our measure can also be used for the development of motion controllers for physics-based characters. More specifically, our measure can be used as an optimization criterion for controllers that need to minimize physical injury, for instance while falling or blocking obstacles.

Our physics-based character model consists of rigid bodies and does not constitute tissue damage. As a result, our model is not suitable for measuring injuries such as gunshots wounds or cuts. The use of our model is limited to injuries from to collisions with large, blunt objects. Examples of such injuries are a character falling to the ground or from a staircase, a character in a vehicle hitting a wall, or a character being hit by a rock with the shape and size of a football.

The remainder of this paper is organized as follows. We start by describing related work on physics-based character animation, injury measurement, and animation perception. We then describe in detail our injury assessment model, including sources for various model parameters. Next, we present methodology and results of the user study conducted to investigate the correlation with perceived injury levels. We conclude with a discussion and pointers for future research.

\section{Related Work}

Physics-based Character Animation After a long period of focus on datadriven animation, the topic of physics-based character animation has recently been subject to renewed interest [1]. Many recent attempts have resulted in impressive behaviors, such as flexible and robust walking control [2], agile databased locomotion control [3], and controllers for traversing uneven terrain [4, 5]. For an overview of relevant aspects, approaches and techniques, we refer to [6]. Physics-based animation research that explicitly deals with injury is limited, although several papers demonstrate its potential in that field $[7,8]$. Parameter optimization (one possible use for our injury measure) has been shown to lead to interesting behaviors [9-11]. For example, Wang et al. [11] show how walking controllers can be made more robust by optimizing for random external perturbations.

Injury Assessment There exists a long history of research on human injury assessment, most of which is based on statistical data from real-life injuries [12]. For the development of our measure we focus on research on injuries associated 
with motor-vehicle accidents. The Abbreviated Injury Scale (AIS) is a measure developed by the Association for Advancement of Automotive Medicine as a system for rating injuries in motor-vehicle accidents [13]. It consists of a dictionary of injury descriptions (mostly related to tissue or bone damage) and associated severity scores, and has been updated several times since its introduction. In 1984, General Motors published a set of reference values intended as guidelines for injury potential [14], based on experiments with the Hybrid III crash test dummy [15], which has been developed to represents the 50-th percentile male adult. Each of these Injury Assessment Reference Values (IARVs) refers "to a human response level below which a specific significant injury is considered unlikely to occur for a given individual," which corresponds roughly with AIS $\geq 3$. Most of these values consist of physical measurements and have a direct equivalent in physics simulation. Several later publications contain similar values for various body parts [16-22]. Both the AIS and IARV focus on injuries to individual body parts. An adapted scale, called the (New) Injury Severity Score (ISS or NISS) defines how to combine IAS values from multiple traumas [23, 24].

Animation Perception Since the perception of injury is an important aspect of our research, it is also relevant to mention research on animation perception. Most well-known is the work of the group of O'Sullivan, including recent publications on plausibility of animated group conversations [25], and on perception of animations subject to time-warping [26]. Studies on physical reality of character animation include the work of Reitsma and Pollard [27], who observe through user studies that errors in horizontal velocity and added accelerations in human jumping motions are easier observed by humans than errors in vertical velocity and added decelerations. Geijtenbeek et al. [28] develop a method to evaluate the physical realism of animations using a musculoskeletal model developed for research in biomechanics.

\section{Injury Assessment Model}

In this section we describe how our injury measures are computed, using values from physical simulation. We will describe the general form of each measure, how each individual measure is computed, and how the individual measures can be combined into a single measure representing total injury.

\subsection{Overview}

Our injury assessment model consists of a set of individual measures, all of which represent an IARV-normalized maximum of a simulated variable averaged over a specific time window. The size of this time window is important, since the maximum of simulated variables such as acceleration can become very high in rigid body simulation. Luckily, most referenced research papers mention appropriate time windows. An overview of all measures is shown in Table 1. 


\begin{tabular}{c|c|c|c|c|c} 
Region & Physical Property & Unit & Window $(\boldsymbol{w})$ & IARV $(\boldsymbol{c})$ & Measure \\
\hline Head & Linear Acceleration [16] & $\mathrm{HIC}$ & 0.015 & 700 & $D_{H}$ \\
Neck & Axial Compression [14] & $\mathrm{kN}$ & 0.030 & 4.0 & $D_{N C}$ \\
Neck & Axial Tension [14] & $\mathrm{kN}$ & 0.035 & 3.3 & $D_{N T}$ \\
Chest & Thoracic spine acceleration [16] & $G$ & 0.003 & 60 & $D_{C}$ \\
Pelvis & Pelvis Acceleration [17, 18] & $G$ & 0.003 & 130 & $D_{P E}$ \\
Arms & Compressive Wrist Loading [22] & $\mathrm{kN}$ & $0.010^{*}$ & 8.0 & $D_{W_{L}}, D_{W_{R}}$ \\
Legs & Femur Axial Force [14] & $\mathrm{kN}$ & 0.010 & 9.1 & $D_{F A_{L}}, D_{F} A_{R}$ \\
Legs & Femur Planar Force [19] & $\mathrm{kN}$ & 0.003 & 3.9 & $D_{F P_{L}}, D_{F P_{R}}$ \\
Legs & Tibia Axial Force [20] & $\mathrm{kN}$ & $0.010^{*}$ & 8.0 & $D_{T_{L}}, D_{T_{R}}$ \\
Legs & Compressive Ankle Loading [14] & $\mathrm{kN}$ & $0.010^{*}$ & 8.0 & $D_{A_{L}}, D_{A_{R}}$ \\
Feet & Foot Acceleration [21] & $G$ & $0.010^{*}$ & 150 & $D_{P_{L}}, D_{P_{R}}$
\end{tabular}

Table 1. Overview of injury measures for individual body regions. *Marked window sizes indicate estimates.

Since all measures have a similar form (with the exception of the measure for head injury), we define the following function to simplify subsequent definitions:

$$
D(v(t), w, c)=\left\{\int_{t_{0}}^{t_{0}+w} \frac{1}{c w} v(t) d t\right\}_{\max }
$$

where $w$ is the window length, $c$ is the IARV value used for normalization, and $t_{0}$ is a starting time of a time window instance. $D(v(t), w, c) \rightarrow[0, \infty\rangle$ represents the normalized maximum of the $w$-sized window-average of physical property $v(t)$. A value of 1 represents the threshold of significant injury.

\subsection{Individual Injury Measures}

Head A common measure for head injury is the Head Injury Criterion (HIC), which is based on linear acceleration of the head [16]. This measure differs slightly in form from our previously defined $D(v(t), w, c)$, since it is based on a non-linear relationship:

$$
H I C(w)=\left\{\left[\frac{1}{w} \int_{t_{0}}^{t_{0}+w}\left\|a_{H}(t)\right\| d t\right]^{2.5} w\right\}_{\max }
$$

in which $w$ is the window time and $a_{H}(t)$ is the acceleration of the head at time $t$. A common window time is $15 \mathrm{~ms}$ (also referred to as the HIC-15 score), and corresponding IARV is 700 [16], which results in head injury measure $D_{H}$ :

$$
D_{H}=\frac{1}{700} H I C(0.015)
$$


Neck Our neck injury measure is based on studies evaluating neck compression and tension [14]. To measure these properties, we regard the axial constraint forces that are applied by the neck joint to the head and chest joint. If $\hat{F}_{H}(t)$ is the constraint force applied to head, and $\hat{F}_{C}(t)$ the constraint force applied to the chest, then the neck injury measures $D_{N C}$ and $D_{N T}$ can be acquired using the dot product of each force and the Y-axis of the neck joint, $y_{N}$ (see Figure $2)$, all in the global coordinate frame:

$$
\begin{aligned}
D_{N C} & =D\left(\max \left(\hat{F}_{H}(t) \cdot y_{N}, 0\right)-\min \left(\hat{F}_{C}(t) \cdot y_{N}, 0\right), 0.030,4.0\right) \\
D_{N T} & =D\left(\max \left(\hat{F}_{C}(t) \cdot y_{N}, 0\right)-\min \left(\hat{F}_{H}(t) \cdot y_{N}, 0\right), 0.035,3.3\right)
\end{aligned}
$$

Chest Our chest injury measure is based on the maximum acceleration of the thoracic spine [16]. We acquire chest injury measure $D_{C}$ by using the acceleration of the chest segment of our virtual character, $a_{C}(t)$ :

$$
D_{C}=D\left(\left\|a_{C}(t)\right\|, 0.003,60\right)
$$

Pelvis Our pelvis injury measure is based on the maximum acceleration the pelvis during side impact, based on biomechanics research by Morgan et al. [17] and Janssen et al. [18]. We acquire our pelvis injury measure $D_{P E}$ using the acceleration of the virtual pelvis segment, $a_{P E}(t)$ :

$$
D_{P E}=D\left(\left\|a_{P E}(t)\right\|, 0.003,130\right)
$$

Arms Muller et al. [22] investigate maximum force that can be applied to the wrist before fracture. To acquire wrist injury $D_{W}$, we use the total magnitude of the constraint forces applied by the wrist joint to hand $\hat{F}_{H A}(t)$ and lower arm $\hat{F}_{L A}(t)$. Muller et al. mention no time window; based on averages from Mertz et al. [14] we decided upon using a $10 \mathrm{~ms}$ window. The measure then becomes (computed separately for left wrist, $D_{W_{L}}$, and right wrist, $D_{W_{R}}$ ):

$$
D_{W}=D\left(\left\|\hat{F}_{H A}(t)\right\|+\left\|\hat{F}_{L A}(t)\right\|, 0.010,8.0\right)
$$

Legs There exist several different publications on IARV scores for legs. These include values for femur axial force $\left(F_{F A}\right)$ [14], femur planar force $\left(F_{F P}\right)$ [19] and tibia axial force $\left(F_{T A}\right)$ [20]. We acquire these forces by taking the magnitude of the acceleration of the corresponding virtual body segment in a specific direction in the local coordinate frame, multiplied by the body segment mass. The resulting injury measures $\left(D_{F A}, D_{F P}\right.$ and $\left.D_{T}\right)$ then become (individually for left and right leg): 


$$
\begin{aligned}
D_{F A} & =D\left(\left\|F_{F A}(t)\right\|, 0.010,9.1\right) \\
D_{F P} & =D\left(\left\|F_{F P}(t)\right\|, 0.003,3.9\right) \\
D_{T} & =D\left(\left\|F_{T A}(t)\right\|, 0.010,8.0\right)
\end{aligned}
$$

Ankles Mertz [14] has published data on maximum ankle load. We compute the corresponding ankle damage score, $D_{A}$, similar to wrist damage, using constraint forces on lower leg, $\hat{F}_{T A}(t)$, and foot, $\hat{F}_{P}(t)$ (individually for left and right):

$$
D_{A}=D\left(\left\|\hat{F}_{T A}(t)\right\|+\left\|\hat{F}_{P}(t)\right\|, 0.010,8.0\right)
$$

Feet Zeidler [21] has suggested the IARV score for foot acceleration, $a_{P}(t)$, to be $150 G$. We again use an estimated window size of $10 \mathrm{~ms}$ to compute our foot injury measure $D_{P}$ (individually for left and right foot):

$$
D_{P}=D\left(\left\|a_{P}(t)\right\|, 0.010,150\right)
$$

\subsection{Combining Individual Measures}

We have so far defined a set $\mathcal{D}$ of individual injury measures, each describing the amount of injury of a specific body part (see Table 1). Since each value is normalized to represent overall severity (with a value of 1 representing the threshold of serious injury), we can meaningfully combine the individual measures by using the average, $D_{A V G}$ :

$$
D_{A V G}=\sum_{D \in \mathcal{D}} \frac{1}{\|\mathcal{D}\|} D
$$

Alternatively, one can choose to only use the three highest scores for this average, inspired by the New Injury Severity Score (NISS) [24], or to use a quadratic relation, as suggested by [23]. However, our later results do not support the idea this will lead to better estimates of perceived total injury.

\section{Experimentation}

To evaluate our injury assessment model, we have conducted a user study to investigate if the output of our model correlates with injury levels of virtual characters as perceived by human observers. This section describes the experiment setup and results. 


\subsection{Trial Data}

Our trial data consisted of 30 short ( 4 second) video clips of a virtual character falling in a physically simulated environment. In each trial, the virtual character falls down from a random initial orientation and height. The initial orientation was generated by applying random rotations around the $\mathrm{X}$ and $\mathrm{Z}$ axis, while the initial height of the center of mass $(\mathrm{COM})$ was varied between $1.5 \mathrm{~m}$ and $4.5 \mathrm{~m}$. An example fragment of a trial video clip is shown in Figure 1.
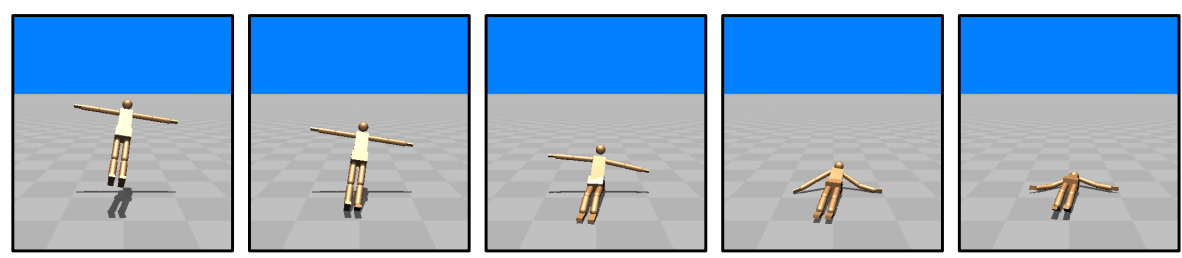

Fig. 1. Example fragment of animation sequence shown as part of the survey.

We used the Open Dynamics Engine (ODE) [29] to perform the physics simulation that generated the animation, using an integration time step of $0.0003 \mathrm{~s}$. We utilized ODE's Error Reduction Parameter (ERP) and Constraint Force Mixing parameter (CFM) to emulate the effect of shock absorption due to soft tissues, joint compliance and ligaments $(\mathrm{ERP}=0.5, \mathrm{CFM}=0.001$; see [29] for details). The hierarchy and degrees-of-freedom (DOFs) of our virtual character are shown in Figure 2. Low-gain PD-controllers were used to drive the character joints towards their initial T-stance position $\left(k_{p}=10, k_{d}=2\right)$, with torque maximum set to $1000 \mathrm{Nm}$ for each joint.

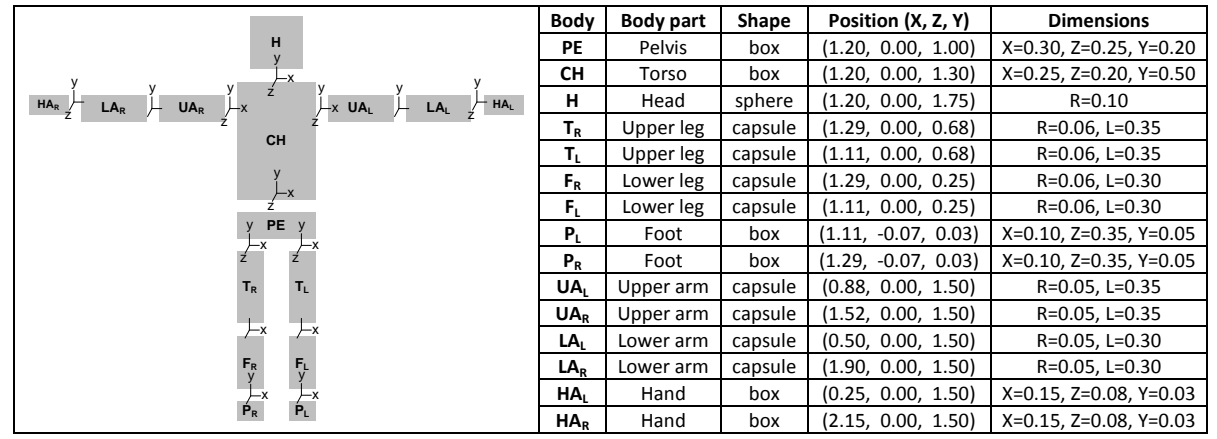

Fig. 2. Character DOFs, body segments, and corresponding dimensions. 


\subsection{User Study}

The trial data was presented to 34 individuals (21 male, 13 female), with ages between 23 and 35 years. Each individual was shown all clips in random order; they were instructed to assign each clip a value between 1 (minimum) and 7 (maximum), based on how much total physical damage they felt was inflicted upon the virtual character. Furthermore, they were informed that a score of 4 represents the threshold of significant injury. This resulted in a total of $N=$ $34 \times 30=1020$ evaluations.

\subsection{Results}

We have found a significant correlation between injury measure $D_{A V G}$ and the corresponding user score $S: \operatorname{corr}\left(D_{A V G}, S\right)=0.603$, with $N=1020$ and $p<$ 0.001. Figure 3 displays a scatter plot of the computed $D_{\text {avg }}$ for each trial, and the corresponding averages of user score $S$. In comparison, the correlation between initial COM height $H$ and user score $S$ is $\operatorname{corr}(H, S)=0.466$, with $N=1020$ and $p<0.001$. This supports the hypothesis that our measure is a more accurate representation of perceived injury than falling height, when regarding characters falling. An overview of the average of $D_{A V G}$ for each individual user score is shown in Figure 4, affirming the correlation between the two.

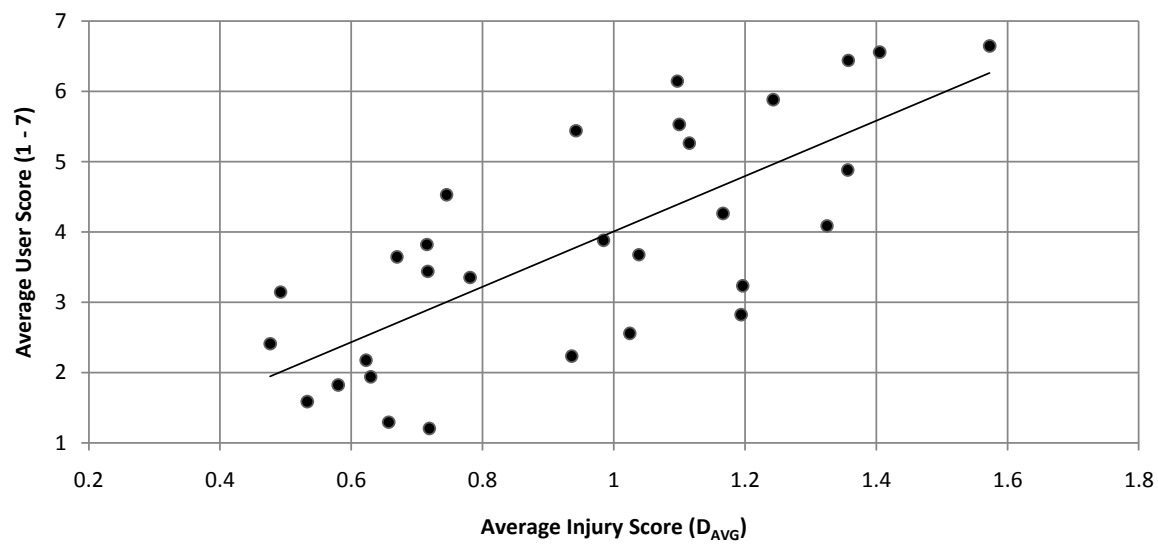

Fig. 3. Scatter plot of injury measure $D_{A V G}$ for each trial (horizontal) and the average of user score $S$ (vertical). $\operatorname{corr}\left(D_{A V G}, S\right)=0.603, N=1020, p<0.001$

If we only regarded the top 3 most occurring injuries (similar to the NISS scoring system), the correlation decreases to $0.522(p<0.001)$. The use of quadratic terms (as in the NISS scoring) only further decreases the correlation. This means our results do not support the use of NISS-related methods for computing overall injury. 


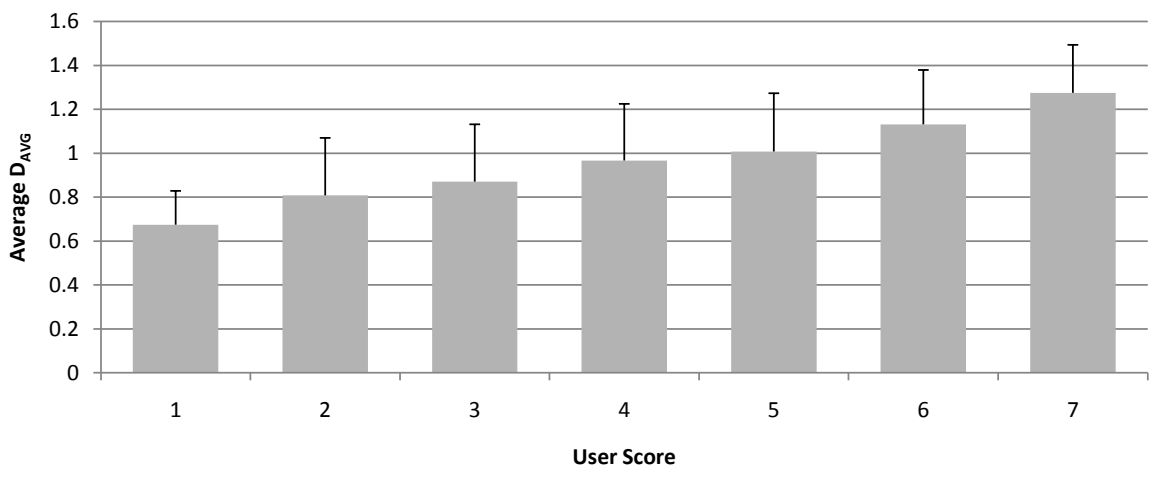

Fig. 4. Average of injury measure $D_{A V G}$ for each user score.

Figure 5 shows the average value for each of the individual measures. It can be seen that neck compression injury $D_{N C}$ is the measure with the highest average score. This is mostly due to the fact that some trials consisting of head-first landing animations had extremely high neck compression. This also explains the high standard deviation of $D_{N C}$ in Figure 4.

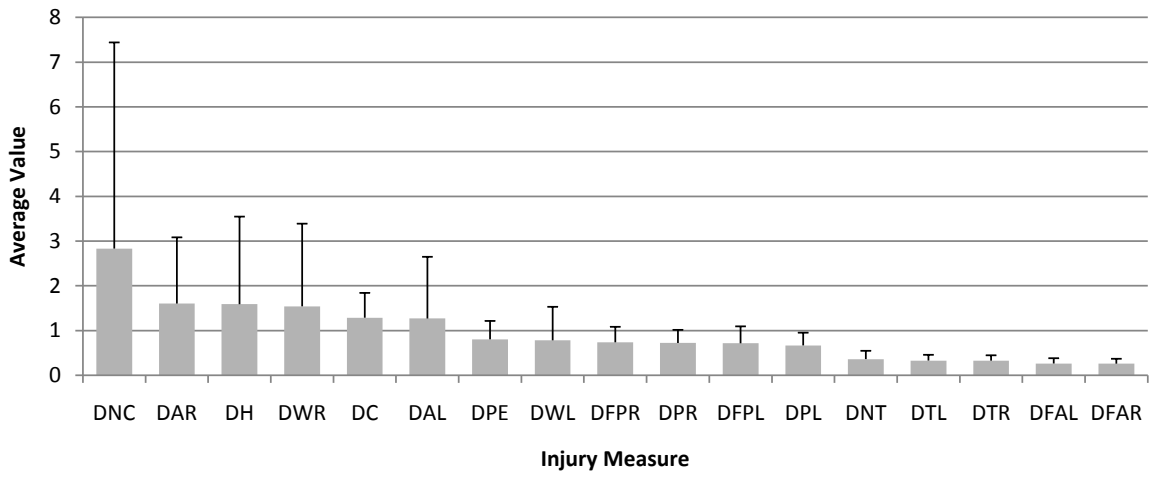

Fig. 5. Average value for each individual injury measure. A value of 1 represents the threshold of serious injury for a specific body part.

Results on correlation studies for individual injury measures show that most measures have a significant positive correlation with perceived injury, while some demonstrate a stronger correlation than others. Measures for ankle compression $\left(D_{A_{L}}\right.$ and $\left.D_{A_{R}}\right)$ show a negative correlation. This can be explained by the fact that ankle compression is highest when a character lands on his feet, while observers generally assigned relatively low injury scores to such animations. 


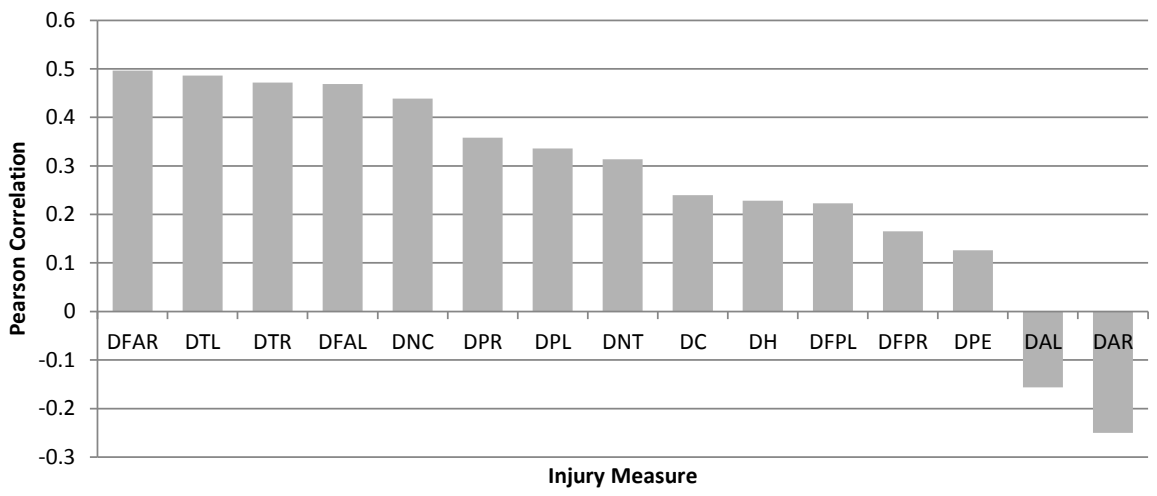

Fig. 6. An overview of the correlations between individual injury measures and perceived injury by human observers. All correlations are significant $(p<0.001)$ except the correlations for wrist injury $\left(D_{W_{L}}\right.$ and $\left.D_{W_{R}}\right)$, which are excluded from the figure.

\section{Conclusion, Discussion and Future Work}

\subsection{Conclusion}

We have presented a model for computing injury measures for physics-based characters, based on research on human injury response levels. We have validated this model by demonstrating a significant correlation between the average injury measure and the total injury level as perceived by human observers. This correlation is stronger than the correlation between perceived injury and fall height $(0.603$ versus 0.466 , respectively, with $N=1020$ and $p<0.001)$.

\subsection{Discussion}

There are several limitations to our injury assessment model. Key body parts such as knees, elbows and shoulders are currently missing, because we could not locate any suitable data. However, we do not believe that these omissions have disrupted our research too much, since relevant trials had significant injury in neighboring body parts. If desired, proper estimates may be generated based on other measures, without the need for published data. It may be possible to assess specific facial injuries by using location-aware impact measures.

In order to measure injury types such as shot wounds or cuts, we suggest to use different approach than physical modeling. Even though it may be possible to create a model for tissue damage, it is questionable whether this is worth the effort for gaming applications.

Perception studies often leave room for debate, and ours is no different. First, we used a very basic looking character model; a more detailed model may have triggered different responses. Second, we have only tested for straight falling 
motions; other motions (falling from stairs, colliding with objects, etc.) may also have triggered different responses.

Several of our test subjects reported that some animations appeared unrealistic, because characters bounced where they normally would break (we did not allow any joint dislocation in our simulation). There are many additional appearance factors that complicate our perception study, such as viewing angle, playback speed, character model, background color, etc. However, test results indicate our test material was at least partly representative.

\subsection{Future Work}

The work presented in this paper is an initial attempt at injury assessment for physics-based virtual characters. There is much room for improvement, while additional user studies can create better insights on how injury of animated characters is perceived. As mentioned in our introduction, injury is an important aspect of gaming, which validates further research in this area.

\section{Acknowledgments}

This research has been supported by the GATE project, funded by the Netherlands Organization for Scientific Research (NWO), as well as the Dinu Patriciu Foundation.

\section{References}

1. Aaron Hertzmann and Victor Zordan. Physics-Based Characters. Computer Graphics and Applications, 31(4):20-21, 2011.

2. S. Coros, P. Beaudoin, and M. van de Panne. Generalized biped walking control. ACM Transactions on Graphics (SIGGRAPH), 29:1-9, 2010.

3. Uldarico Muico, Yongjoon Lee, Jovan Popović, and Zoran Popović. Contact-aware nonlinear control of dynamic characters. ACM Transactions on Graphics (SIGGRAPH), 28(3):1-9, July 2009.

4. Jia-chi $\mathrm{Wu}$ and Zoran Popovic. Terrain-Adaptive Bipedal Locomotion Control. ACM Transactions on Graphics (SIGGRAPH), 29(4):1-10, 2010.

5. I. Mordatch, M. de Lasa, and A Hertzmann. Robust Physics-Based Locomotion Using Low-Dimensional Planning. ACM Transactions on Graphics (SIGGRAPH), 29(4):1-8, 2010.

6. P. Faloutsos. The virtual stuntman: dynamic characters with a repertoire of autonomous motor skills. Computers \& Graphics, 25(6):933-953, December 2001.

7. Y. Abe and M da Silva. Multiobjective control with frictional contacts. Proc. of the 2007 ACM SIGGRAPH/Eurographics symp. on Computer animation, pages 249-258, 2007.

8. Karl Sims. Evolving virtual creatures. Proc. of the 21st annual conf. on Computer graphics and interactive techniques - SIGGRAPH '94, pages 15-22, 1994.

9. J.M. Wang, D.J. Fleet, and A. Hertzmann. Optimizing walking controllers. In ACM Transactions on Graphics (TOG), page 168. ACM, 2009. 
10. JM Wang, DJ Fleet, and A Hertzmann. Optimizing Walking Controllers for Uncertain Inputs and Environments. ACM Transactions on Graphics (SIGGRAPH), 29(4):1-8, 2010.

11. E. Petrucelli. The abbreviated injury scale: Evolution, usage and future adaptability. Accident Analysis \& Prevention, 13(1):29-35, March 1981.

12. The Abbreviated Injury Scale, 1990 Revision. Technical report, Association for the Advancement of Automotive Medicine, Morton Grove, Illinois, 1990.

13. H.J. Mertz. Injury assessment values used to evaluate Hybrid III response measurements. NHTSA docket, 74:14, 1984.

14. J.K. Foster, J.O. Kortge, and M.J. Wolanin. Hybrid III-a biomechanically-based crash test dummy. Technical report, Society of Automotive Engineers, 400 Commonwealth Dr, Warrendale, PA, 15096, USA, 1977.

15. H.J. Mertz, P. Prasad, and A.L. Irwin. Injury risk curves for children and adults in frontal and rear collisions. Technical report, Soc. of Automotive Engineers, 1997.

16. R.M. Morgan, J. Marcus, and R. Eppinger. Side impact-the biofidelity of NHTSA's proposed ATD and efficacy of TTI. SAE Tech. Pap. Ser, 1986.

17. E. G. Janssen, J. Wismans, and P. J. A. de Coo. Comparison of Eurosid and Cadaver Responses in Side Impact. In Twelfth International Technical Conference on Experimental Safety Vehicles, 1989.

18. J.R. Kerrigan, K.S. Bhalla, J.R. Funk, N.J. Madeley, and D. Bose. Experiments for Establishing Pedestrian-Impact Lower Limb Injury Criteria. SAE Technical Paper, 2003.

19. JR Crandall, SM Kuppa, GS Klopp, GW Hall, WD Pilkey, and SR Hurwitz. Injury mechanisms and criteria for the human foot and ankle under axial impacts to the foot. International Journal of Crashworthiness, 3(2):147-162, 1998.

20. F. Zeidler. The significance of lower limb injuries of belted drivers. Journal of Orthopedics, 1984.

21. M. E. Muller, C. E. Webber, and M. L. Bouxsein. Predicting the failure load of the distal radius. Osteoporosis International, 14(4):345-52, June 2003.

22. S.P. Baker, B. O'Neill, W. Haddon Jr, and W.B. Long. The injury severity score: a method for describing patients with multiple injuries and evaluating emergency care. The Journal of trauma, 14(3):187, 1974.

23. M Stevenson, M Segui-Gomez, I Lescohier, C Di Scala, and G McDonald-Smith. An overview of the injury severity score and the new injury severity score. Injury Prevention, 7(1):10-3, March 2001.

24. C. Ennis, R. McDonnell, and C. O'Sullivan. Seeing is believing: Body motion dominates in multisensory conversations. ACM Transactions on Graphics (TOG), 29(4):1-9, 2010.

25. M. Pražák, R. McDonnell, and C. O'Sullivan. Perceptual evaluation of human animation timewarping. In ACM SIGGRAPH ASIA 2010 Sketches, page 30. ACM, 2010.

26. PSA Reitsma. Perceptual metrics for character animation: sensitivity to errors in ballistic motion. ACM SIGGRAPH 2003 Papers, 22(3):537-542, 2003.

27. T. Geijtenbeek, A. van den Bogert, B. van Basten, and A. Egges. Evaluating the physical realism of character animations using musculoskeletal models. In Motion in Games, Lecture Notes in Computer Science, pages 11-22, Berlin, Heidelberg, 2010. Springer.

28. Russell Smith. Open Dynamics Engine User Guide v0.5, 2006. 